\title{
Molecular insights of melioidosis causing Burkholderia pseudomallei strains in Sri Lanka
}

\author{
A. W. A. Chathura Wikumpriya Gunasekara, LGTG Rajapaksha \\ Veterinary Medical Center and College of Veterinary Medicine, Jeonbuk National University, Jeonju, South Korea \\ Two authors equally contributed to this work
}

\begin{abstract}
Objectives: Objectives: Gram-negative pathogenic bacterium Burkholderia pseudomallei is the causative organism of melioidosis, predominantly reported in Southeast Asia. The infections in humans can be recurrent, and sometimes difficult to cure. Studying the genome of $B$. pseudomallei is the key to understand origins, transmission routes, and phylogenetic relationships.
\end{abstract}

Methods: We compared all available B. pseudomallei genomes from the NCBI database representing Sri Lanka to 15 previously reported genomes in Asia. The analysis involved in silico MLST, wgMLST, single nucleotide polymorphism (SNP), average nucleotide identity (ANI), clonal complexes (CC), virulence, and antibiotic resistance profiles.

Results: The MLST analyses of 24 strains revealed, 6 Sri Lankan and 1 Indian strain formed CC594*, a novel single locus variant clonal complex, and 3 strains from Malaysia, Vietnam, Thailand formed another clonal complex named CC70*. From 9 Sri Lankan strains, BPs122 and BPs133 had ancestral origins tied to BPs114 with 114/99.6\% and 140/99.6\% for SNPs/ANIs. In CC70*, Thailand and Vietnam strains had 1196/99.95\% for SNPs/ANIs, respectively. Among the Sri Lankan strains, actin-based motility gene bimA detected in BPs110 only, whereas LPS antigen was presented in BPs112, BPs115, and BPs116 genomes. A total of 67 genes related to antibiotic resistance (22 multidrug efflux systems, 10 regulators modulating/expression of antibiotic resistance, and 11 antibiotic inactivation enzymes related genes) were identified.

Conclusion: The B. pseudomallei strains in Sri Lanka represent a highly diverse nature and some of them had clonal relationships with other Asian strains. The present study concludes B. pseudomallei strains in Sri Lanka have probably risen from different ancestral origins. J Microbiol Infect Dis 2020; 10(4): 215-221.

Keywords: Burkholderia pseudomallei, Sri Lanka, SNPs, ANIs, clonal relation

\section{INTRODUCTION}

The intracellular facultative gram-negative pathogenic bacterium Burkholderia pseudomallei is the etiological agent of lifethreatening infectious disease melioidosis. This bacterium can inhibit a wide range of environments including soil, contaminated water, single-cell microbes, plants, and animals $[1,2]$. To date, melioidosis infections are prominent in Southeast Asia and Northern Australia [3]. A recent study predicted that Southeast Asia has the highest prevalence of melioidosis cases (44\%) compared to other regions [4]. In humans, melioidosis is found to be correlated with other factors such as diabetes mellitus, immunosuppression, chronic kidney disease, and excessive alcohol intake. So far, no vaccines have been developed to counter the disease, and B. pseudomallei is shown to be intrinsically resistant to many antibiotics $[5,6]$. Recently, several pathogenesis factors including capsules, quorum sensing, and protein secretion systems (type I, II, V, and 3 different T3SS1 systems) have been identified from the $B$. pseudomallei genome [7]. Moreover, the incubation period of the bacterium can be varying from 2 days to months or years. Hence, in some cases, the disease was found to be recurrent with the reactivation of latent infection. Today, standard guidelines for therapy had

Correspondence: Dr. A. W. A. C. W. R. Gunasekara, Veterinary Medical Center and College of Veterinary Medicine, Jeonbuk National University, 54596, Jeonju, South Korea Email: cwikumpr@jbnu.ac.kr 
produced better results on reducing mortalities and preventing recurrences of the disease.

In terms of the tropical country, Sri Lanka is located near the equator where melioidosis is predominant [8]. This country was the first to report melioidosis (in 1927) from the South Asian region. In 2004 the disease reemerged from an Australian tourist who visited Sri Lanka, and in 2005 the first case of melioidosis from a Sri Lankan person was reported [9]. Since then the disease started to emerge frequently and around 250 additional cases were confirmed from 2006 to 2017 from all the provinces of Sri Lanka, whereas the majority were associated with other risk factors such as diabetics and thalassemia while most of the infected people belonged to the agricultural communities [9]. Moreover, it is reported that the presence of the disease in rural communities goes undetected most of the time, and this suggests the infection with $B$. pseudomallei can be more prevalent in Sri Lanka than previously recognized [8].

With the development of sequencing technology, whole-genome sequencing (WGS) became very popular and different WGS methods have been successfully adopted to identify and trace the origin of different bacterial isolates in many pathological fields. The use of WGS utilizes single nucleotide polymorphisms (SNP) identified from the genomes and it assists to confirm the epidemiological relationships of bacterial isolates $[10,11]$. To date, many studies have been conducted to identify the occurrence of melioidosis causing $B$. pseudomallei in Sri Lanka. As a result, in 2019 the first 8 wholegenomes of $B$. pseudomallei strains representing Sri Lanka have been deposited [12]. However, an in-depth investigation into their evolutionary origins related to Asian strains has not been studied so far.

In the present study, the research question was whether the epidemiological origins of melioidosis causing Sri Lankan B. pseudomallei strains are related to other Asian strains? To answer this question, a bioinformatics analysis based on in silico MLST (multilocus sequence typing), wgMLST (whole-genome multilocus sequence typing), core genome single nucleotide polymorphisms (SNPs), average nucleotide polymorphism (ANIs), virulence, and antibiotic-related genes was conducted. The in- depth genomic analysis performed here is the first whole genome-scale insight into the diversity and/or similarities of Sri Lankan $B$. pseudomallei strains.

\section{METHODS}

\section{Establishment of Sequence database for the study}

A total of 24 melioidosis causing $B$. pseudomallei strains from the NCBI database were used for the study. The established database consisted of genomes from Asian countries, including India (3), Pakistan (1), Sri Lanka (9), Thailand (2), Taiwan (2), Malaysia (3), Vietnam (1), China (2). The strain K96243 (isolated from Thailand in 1996) was used as the reference genome for this study. Some of the genome sequences of the established database were de novo assemblies. If the SRA reads (raw data) for de novo assemblies available, they were separately analyzed to reconstruct the genomes of representative strains.

\section{In silico MLST and population analysis}

Center for genomic epidemiology online serverbased CGE MLST 2.0, in silico plugin [13] used to confirm the sequence types (STs) of 24 selected strains. The pubMLST database suggested 7 housekeeping genes were used to construct the MLST phylogenetic tree and the evolutionary relationships were analyzed using the kimura-2 parameter method in MEGA genomic software. The statistical support of the nodes in the phylogenetic tree was assessed by setting the bootstrap value to 1000 . There was a total of 3401 positions in a single concatenated genomic sequence.

Many researchers use clonal complexes (CCs) to study the evolutionary relationships of closely related strains [14]. In the present study, eBURST (V3) program was applied to detect different clonal complexes and the PHYLOViZ online server was used to design the population snapshot for 24 B. pseudomallei strains. The common group definition was used to assess the CCs as two different STs are considered a single locus variant (SLV) when they differ from each other at a single locus. Double locus variants (DLV) are any two different STs differing in two loci. At least six of the seven alleles had to be identical to include in the same group or to a CC. 


\section{Identification of SNPs and ANIs among bacterial strains}

The core genome represents the genes shared by all the strains of a species. Hence, the variations in core genome can provide evidence on evolutionary relationships [15]. In this aspect, core genome SNP and ANI analyses were performed for all the strains in the established database. The ANIs were identified by using the ANI blast online server JSpeciesWS [16]. The SNP calling was performed from a newly designed protocol. First, the core genomes of 24 strains were identified using the BIGSdb genome comparator [17] available in the pubMLST database (https://pubmlst.org/software/database/bigsdb/), by aligning them to the K96243 genome as the reference. The produced core genome sequences were then separated by using the Genious software. Following this, to identify SNPs, CSI Phylogeny 1.4 online server at the Center for genomic epidemiology was used [18] The following criteria were used for high-quality SNP calling and filtering: (I) a minimum depth of $10 \times$ at SNP positions, (II) a minimum relative depth of $10 \%$ at SNP positions, (III) a minimum distance of $10 \mathrm{bp}$ between SNPs, (IV) a minimum SNP quality of $30,(\mathrm{~V})$ a minimum read mapping quality of 25 , and (VI) a minimum Zscore of 1.96. Site validation for each SNP position was performed. The SNPs that fail the requirements were excluded from the final analysis. Based on concatenated alignments of high-quality SNPs, maximum likelihood trees were created using Fast Tree version 2.1.7.

\section{Whole-genome phylogenetic analysis}

Phylogenetic analysis was performed for $B$. pseudomallei strains using the wgMLST comparator tool available on the pubMLST website. The produced loci were categorized into 3 groups as 1) variable in the isolates, 2) identical in the isolates, 3 ) missing or incomplete due to being located at the ends of contigs. The loci of "variable and identical in all isolates" was used to analyze their relationships in the established database, producing a distance matrix based on the number of loci. The neighbor-net algorithm was used to map the distance matrix of these loci and it was analyzed through Split tree (V4) software.

\section{Antimicrobial resistance genes and Virulence factors}

PATRIC genomic database (https://www.patricbrc.org/) was applied to identify antibiotic resistance genes among Sri Lankan B. pseudomallei strains [19]. The presence of common antibiotic resistance and/or related to confer resistance was analyzed based on the K96243 reference strain. The online server database Virulence Factors of Pathogenic Bacteria (VFDB) was applied for the identification of virulence factors among the strains [20]. In virulence factor analysis genes that are considered important in previous studies for the pathogenicity of $B$. pseudomallei were studied.

\section{RESULTS}

\section{MLST analysis and Assignment to clonal complexes}

The MLST analysis based on 7 housekeeping genes (ace, gltB, gmhD, lepA, lipA, narK, and $n d h)$ confirmed, that $B$. pseudomallei has a highly diverse nature. There was a total of 20 different STs for the 24 strains. Among them, ST594 was the main ST, whereas the common allelic frequencies were $42 \%, 54 \%, 50 \%, 50 \%$, $88 \%, 42 \%, 58 \%$ for ace (allele- 1 ), gltB (allele- 4 ), gmhD (allele-3), lepA (allele-2), lipA (allele-1), nark (allele-2), and ndh (allele-1) respectively. The MLST phylogenetic tree was divided into 5 cluster groups in which cluster group 1,2 and 3 represented $80 \%, 100 \%$, and $20 \%$ of Sri Lankan strains (Figure 1). Moreover, the evolutionary distance of Sri Lankan strains ranged from $0 \%$ $0.2 \%$. Among them, BPs110 and BPs116 showed the highest evolutionary distance to each other. In comparison, other Asian strains exhibited $0.1 \%-0.5 \%$ evolutionary distances to Sri Lankan strains.

Following MLST analysis, the initial clonal complexes were identified by eBURST software. Then the PHYLOViZ software was used to draw the population snapshot for the 24 strains. The eBURST clonal complex and the population snapshot showed ST594 strains were only different from a single locus (SLV) which shared 6 identical allele types to the strains in ST734, ST1364, ST1413, and ST13. Among them, only ST734 was isolated from India whereas other strains were isolated from Sri Lanka (Figure 2). 
Hence, they are only varied from a single locus this can be considered as a CC, named afterward as CC 594*. Apart from the strains in CC594*, ST70 and ST289 also formed an SLV group named $\mathrm{CC} 70^{*}$. However, interestingly the strains in $\mathrm{CC}^{*}{ }^{*}$ represented 3 different countries namely Thailand (Mahidol106a), Vietnam (NA67), and Malaysia (R15).

\section{Whole-genome phylogenetic analysis}

Whole-genome phylogenetic trees were produced for both chromosomes in response to the reference strain K96243. In the neighbor net phylogenetic tree, chromosome I and chromosome II are composed of 3460 and 2646 alleles respectively. There were 18 lineages in both neighbor net trees. The 3 Sri Lankan strains of BPs114, BPs122, and BPs133 formed a single cluster in both phylogenetic trees as similar to the MLST phylogenetic tree. The close splitting pattern of 3 strains proved they may have originated from a common ancestor not long ago. However, in-depth observation of the neighbor net trees showed that BPs110, BPs111, BPs115, and BPs123 shared the same ancestral roots of this lineage. The strains of BPs115 and BPs123 formed another cluster group after originating from the common ancestral root. But the splitting patterns showed the origin of these two strains possibly have happened a long time ago. Moreover, the strains of BPs116 and BPs112 had separated single lineages from other Sri Lankan strains (Figure $3)$.

In comparison, the strains represented CC70* (Mahidol106a, NA67, and R15) formed another cluster group suggesting the possibility of common ancestral origins. However, the strains in ST70 had a close relationship with each other than the ST289 strain in CC70* group. Moreover, the ST58 and ST55 strains (vgh007, HNB001) formed another cluster group whereas two Malaysian strains of ST1034 (M1 and Ms) formed another. However, in comparison, the neighbor net tree showed Malaysian strains had close ancestral origins than the other two strains.

\section{SNP and ANI analysis}

A total of 9 Sri Lankan B. pseudomallei strains were analyzed for ANIs and SNPs along with 15 other Asian strains. In the results, all Sri Lankan strains had more than $99 \%$ ANI to each other. Among them, the 3 Sri Lankan strains (BPs112, BPs114, BPs133) in CC594* showed the highest ANI (99.96\%), followed by $99.83 \%$ ANI between BPs123 and BPs115. The lowest ANIs were observed as $99.65 \%$ between BPs 110 and BPs123 for Sri Lankan strains. However, among others, the lowest ANI of $98.08 \%$ was observed in between VB2514 and NA67 Asian strains. The SNP analysis showed that the strains clustered as ST594 had 113 (BPs114) and 140 (BPs122) SNPs to the BPs133 strain. The two strains of BPs112 and BPs123 which had a close relationship in the wgMLST phylogenetic tree showed 5044 SNPs to each other (Figure 4). Moreover, the strains of BPs 110 and BPs111 which had the same ancestral roots to CC594* had 6692 and 7040 SNPs respectively. The BPs112 had the highest SNP variations for Sri Lankan strains with more than 10000 SNPs. For other Sri Lankan strains, SNPs ranged from 8000-10000. Furthermore, among the Asian strains, CMManipal had the lowest number of SNPs (10551 SNPs) to the BPs112 Sri Lankan strain. However, among others, the lowest SNPs (89 SNPs) were observed between M1 and Ms Malaysian strains. Very interestingly among the strains in CC70*, NA67 and Mahidoll1106a had 1996 SNPs to each other whereas it was 6174 and 5594 to the R15 Malaysian strain. Moreover, the highest SNP of 15652 was observed in VB2514 and PHLS112 strains.

\section{Antimicrobial resistance genes and Virulence factors}

The antibiotic resistance patterns for Sri Lankan B. pseudomallei strains were studied. There was a total of 67 antibiotic-related genes produced by the PATRIC genomic annotation server. The identified genes were categorized as 22 related to Multidrug efflux systems, 10 related to regulator modulating and expression of antibiotic resistance genes, 11 responsible for antibiotic inactivation enzymes, and 25 for antibiotic target in susceptible species. In contrast all the strains possessed genes which infers the antibiotic resistance for B lactam (penl family), sulfonamide (folP, folA, Dfr), aminoglycosides (oprB family), cefuroxime (rpoC), triclosan (fabV), carbapenem (oprD), isoniazid (katG/katA), fluoroquinolone (gyrA), daptomycin 
(pgsA/gdpD), fosfomycin (murA) and bacitracin (bcrC).

In this study, virulence factors analysis showed unique genomic variations among Sri Lankan $B$. pseudomallei strains. Among them, only BPs 110 strain possessed the gene $\operatorname{bim} A$ responsible for actin-based motility. The adherence factors gene of boaA did not detect in BPs116 whereas boaB gene was observed only in BPs116 and
BPs112. Among the antiphagocytosis related genes, wzt2 was absent in all the tested strains. Moreover, the genes of gmhA and wzm were not presented in the BPs113 strain. In comparison, the LPS antigen was observed only in BPs112, BPs115, and BPs116 strains. Apart from these differences, all the strains had similar gene numbers for invasion (46), quorum sensing (8), and secretion systems (51).

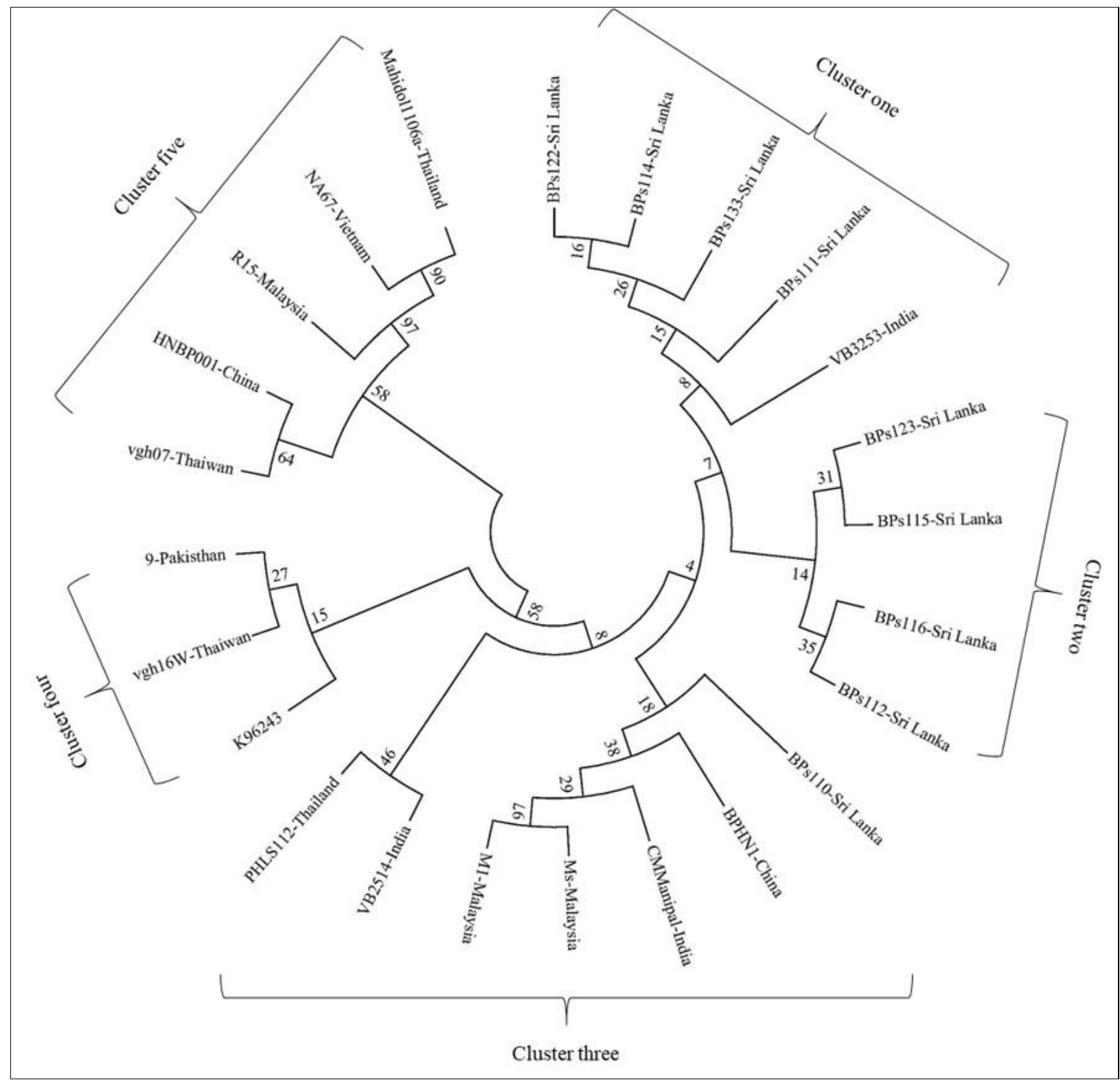

Figure 1. The evolutionary history was inferred by using the Maximum Likelihood method based on the tamura-nei model. The percentage of trees in which the associated taxa clustered together is shown next to the branches. Initial tree(s) were obtained by applying Neighbor-Joining algorithm. The analysis involved 24 nucleotide sequences. All positions containing gaps and missing data were eliminated. There was a total of 3401 positions in the final dataset. Evolutionary analysis was conducted in MEGA7. 


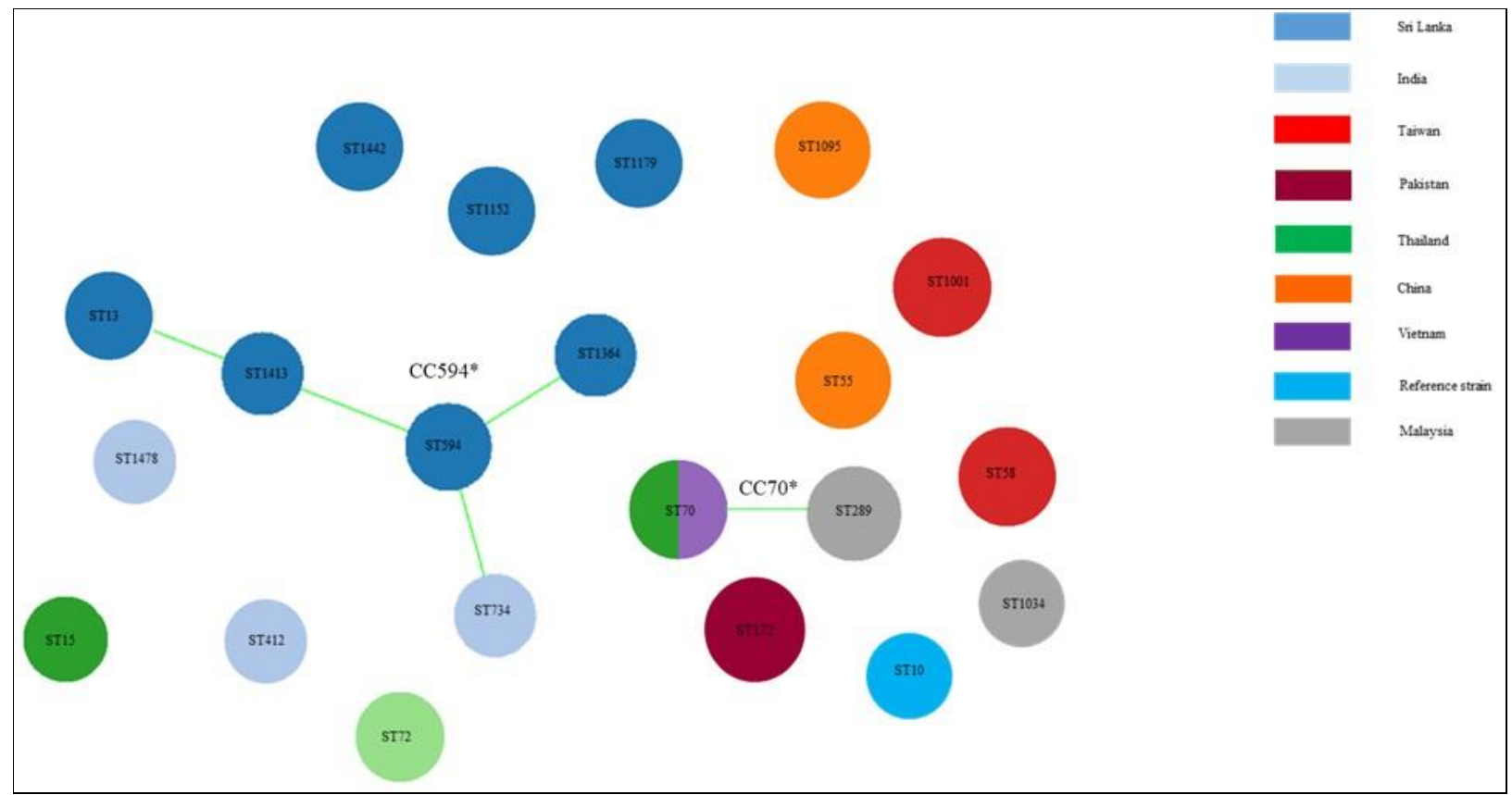

Figure 2. MLST genes-based population snapshot generated by PHYLOViZ online server. Different colors indicate different countries of $B$. pseudomallei strains. Two major clonal groups named as CC594* and CC70* also shown in the population snapshot.

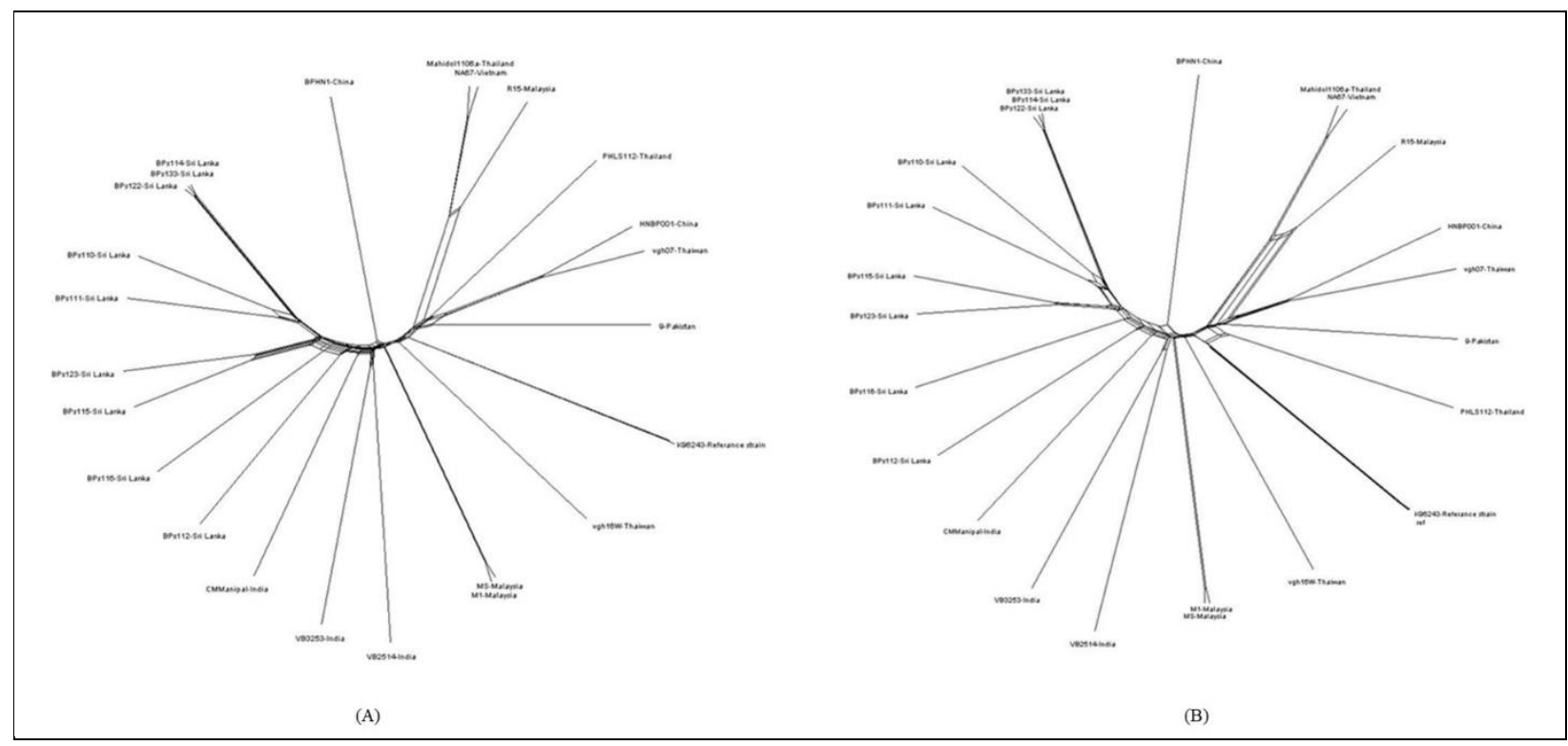

Figure 3. Neighbor-net graph generated by using the BIGSdb Genome Comparator tool within the PubMLST website. (A) 3460 and (B) 2646 loci were identified after using K96243 chromosome I and chromosome II as reference from Genbank. The graph suggests $B$. pseudomallei can undergo evolutionary changes which in return result in high genetic variations. 


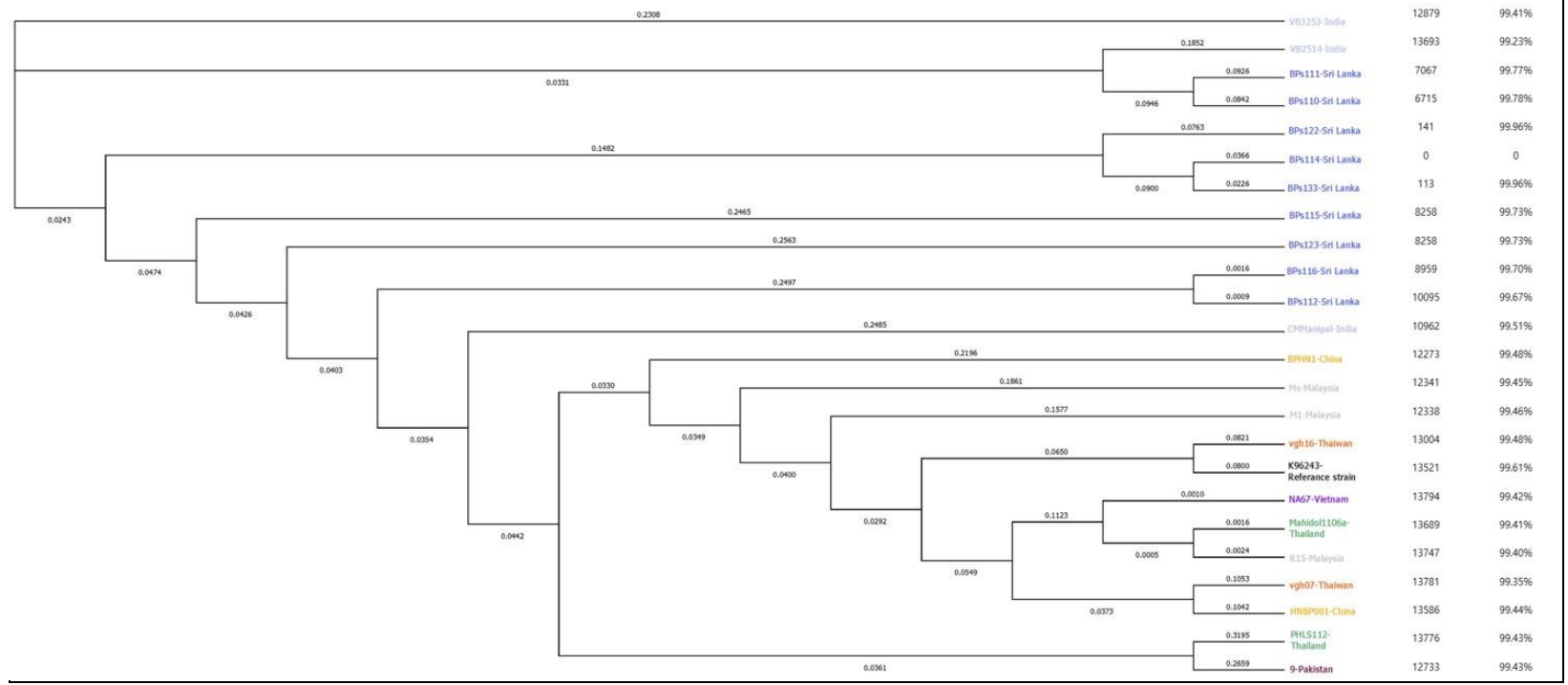

Figure 4. Core genome single nucleotide polymorphism of all the strains. The SNPs and ANIs based on BPs114 strain are indicated in SNP phylogenetic tree. Different colors in the phylogenetic tree indicate different origins in which $B$. pseudomallei strains were isolated.

\section{DISCUSSION}

Burkholderia pseudomallei is a gram-negative bacterium and the causative agent of underrated disease known as melioidosis. In terms of Sri Lanka, melioidosis is shown to be an emerging public health concern. In this aspect, the present study was directed to understand the evolutionary and genomic relationships of Sri Lankan B. pseudomallei strains to other Asian strains. The whole-genome analysis revealed 2 emerging melioidosis clonal complexes in the Asian region. Among them, CC594* is composed of $80 \%$ of Sri Lankan strains. Furthermore, there was a total of 67 genes related to antibiotic resistance in Sri Lankan strains. Among the virulence factors, actin-based motility gene bimA detected only in 1 strain, whereas LPS antigen was observed in 3 Sri Lankan strains.

Many researchers around the world use the pubMLST multilocus sequence typing (MLST) scheme as the common genotyping method to study the genomic relationships among bacterial species. Today the pubMLST database holds 1876 MLST profiles representing B. pseudomallei. However, the MLST scheme is composed of 7 genes only, and it is not enough to represent a large-scale whole-genome, hence not the best tool to understand evolutionary relationships among bacterial strains. In this aspect, investigating WGS associated with $B$. pseudomallei infections can provide more conclusive evidence on genomic relationships even for the strains with the same STs.

In 2009, researchers studied the first known strain of B. pseudomallei and hypothesized it has originated from Australia and possibly transmitted to Southeast Asia in the last glacial period (21). Hence, we can predict all the strains observed in the Asian region (including Sri Lanka) today, may have been originated from Australia and with time have undergone dramatic evolutionary and genetic changes, which in return resulted in differences to its original strains. Despite this fact, close evolution relationships among most recently diverged strains can be studied by using genomic analysis methods such as SNPs, ANIs, and wgMLST. In the present study, 9 Sri Lankan strains formed one major clonal complex based on wgMLST. These strains (BPs122, BP133, BPs114) had close splitting patterns in phylogeny, the lowest SNPs (113,140 and 141), and a single ST (ST594) in MLST, proving the theory of closely related ancestral origins. Interestingly 3 strains from 3 different countries were also clustered together with low SNPs, suggesting possible disease transmission from country to country. However, as a whole, there was a high genetic diversity among $B$. pseudomallei in the Asian region.

In consideration of control and prevention methods, a limited number of antimicrobial agents available for the successful treatment of 
melioidosis. The bacterium B. pseudomallei usually does not possess the common antibiotic resistance mechanisms such as bacterial mobile elements, plasmids, transposons, or integrons, but it inherits resistance through chromosomally encoded genes (28). The antibiotic resistance mechanisms of $B$. pseudomallei related to cell envelope, multidrug efflux systems, and enzymatic inactivation have been reported previously by many other researchers. Moreover, the bacterium was shown to possess many antibiotic resistance genes against common antimicrobial agents (24). In agreement with the above observations, in our study, we were able to identify antibiotic resistance mechanisms and genes responsible for B lactam, sulfonamide, aminoglycosides, cefuroxime, triclosan, carbapenem fluoroquinolone, daptomycin, fosfomycin, and bacitracin resistance from Sri Lankan $B$. pseudomallei strains.

Researchers have identified several virulence factors in B. pseudomallei, including capsular polysaccharide (CPS) and lipopolysaccharide (LPS) involved in opsonophagocytosis inhibition, the Bsa type III secretion system cluster 3 (T3SS-3 related) and cluster 1 type VI secretion system (T6SS-1) that facilitate survival and growth of the organism within host cells, the Actin based mortality and Adherence factors (25-27). In our study, we were able to detect the bimA gene responsible for actin-based motility only in the BPs110 strain which promotes movement both within and between cells. As suggest by other researchers there are other genes such as bimB and bimC also responsible for motility (28). However, in comparison, other Sri Lankan strains did not possess any of the above genes. Among adherence related factors, boaA gene was common, whereas only 2 strains carried boaB gene. However, the boaA gene is also found in $\mathrm{B}$. mallei, but boaB is unique to $B$. pseudomallei as a specific adherence factor (29). Apart from these, the lipopolysaccharide (LPS) is considered as one crucial aspect in eliciting mammalian innate immunity (30). The LPS antigens were only observed in 3 strains namely BPs112, BPs115, and BPs116. However, all other virulence factors were similar among Sri Lankan B. pseudomallei strains.

The major limitation of this study is the availability of WGS representing Asian countries in the NCBI database. We believe large-scale genome sequencing and analysis in the future will be able to provide more information on the transmission pattern of $B$. pseudomallei strains causing melioidosis in Asia.

In conclusion, the present study is the first to analyze Sri Lankan B. pseudomallei strains epidemiological origins, genomic similarities, and variations. There were several distinct lineages among Sri Lankan strains and some of them showed clonal relationships with Low SNPs to other Asian strains. On these points, we can finally conclude B. pseudomallei strains in Sri Lanka has a highly diverse nature and they most probably have risen from several ancestral origins.

\section{ACKNOWLEDGMENTS}

The authors would like to convey their gratitude to the research team (Jayasinghearachchi HS, Corea EM, Krishnananthasivam S, Sathkumara $H D$, Francis VR, Abeysekere TR, De Silva AD, and Jayanthi Masakorala) who primarily conducted the first whole-genome sequencing project for B. pseudomallei isolates in Sri Lanka.

Declaration of Conflicting Interests: The authors declare that they have no conflict of interest.

Funding: The authors received no specific funding for this work.

\section{REFERENCES}

1. Titball RW, Russell P, Cuccui J, et al. Burkholderia pseudomallei: Animal models of infection. Trans $R$ Soc Trop Med Hyg 2008; 102: S111-116.

2. Chewapreecha $C$, Mather AE, Harris SR, et al. Genetic variation associated with infection and the environment in the accidental pathogen Burkholderia pseudomallei. Commun Biol 2019; 22(1):1-11.

3. Cheng AC, Currie BJ. Melioidosis: epidemiology, pathophysiology, and management. Clin Microbiol Rev 2005;18(2):383-416.

4. Mukhopadhyay C, Shaw T, Varghese G, Dance D. Melioidosis in South Asia (India, Nepal, Pakistan, Bhutan and Afghanistan). TropicalMed 2018;22(2):51.

5. Laws TR, Taylor AW, Russell P, Williamson D. The treatment of melioidosis: is there a role for repurposed drugs? A proposal and review. Expert Review of Antiinfective Therapy 2019;17(12):957-967.

6. Massey S, Yeager LA, et al. Comparative Burkholderia pseudomallei natural history virulence studies using an aerosol murine model of infection. Sci Rep 2014;7(1):4305. 
7. Larsen JC, Johnson NH. Pathogenesis of Burkholderia pseudomallei and Burkholderia mallei. Military Medicine 2009;174(6):647-651.

8. Corea E, Thevanesam V, et al. Melioidosis in Sri Lanka: an emerging infection. Sri Lankan J Infec Dis 2012;31(1):2.

9. Corea EM, de Silva AD, Thevanesam V. Melioidosis in Sri Lanka. Trop Med Infect Dis 2018;21(1): PMC6136624.

10. Didelot X, Pang B, Zhou Z, McCann A, Ni P, Li D, et al. The role of china in the global spread of the current cholera pandemic. Casadesús J, editor. PLoS Genet 2015;13(3): e1005072.

11. Fu S, Tian H, Wei D, Zhang X, Liu Y. Delineating the origins of Vibrio parahaemolyticus isolated from outbreaks of acute hepatopancreatic necrosis disease in Asia by the use of whole genome sequencing. Front Microbiol 2017;28(8):2354.

12. Jayasinghearachchi HS, Corea EM, et al. Wholegenome sequences of eight clinical isolates of Burkholderia pseudomallei from melioidosis patients in Eastern Sri Lanka. Maresca JA, editor. Microbiol Resour Announc 2019;8(33): MRA.00645-19, e0064519

13. Larsen MV, Cosentino S, et al. Multilocus sequence typing of total-genome-sequenced bacteria. J Clin Microbiol 2012;50(4):1355-1361.

14. Francisco AP, Bugalho M, Ramirez M, Carriço JA. Global optimal eBURST analysis of multilocus typing data using a graphic matroid approach. BMC Bioinformatics 2009;10(1):152.

15. Stoneking M. Single nucleotide polymorphisms. From the evolutionary past. Nature 2001;409(6822):821-822.

16. Richter M, Rosselló-Móra R, Oliver Glöckner F, Peplies J. JSpeciesWS: a web server for prokaryotic species circumscription based on pairwise genome comparison. Bioinformatics 2016;32(6):929-931.

17. Jolley KA, Bray JE, Maiden MCJ. Open-access bacterial population genomics: BIGSdb software, the PubMLST.org website and their applications. Wellcome Open Res 2018;3: PMC6192448.

18. Kaas RS, Leekitcharoenphon $\mathrm{P}$, Aarestrup FM, Lund $\mathrm{O}$. Solving the problem of comparing whole bacterial genomes across different sequencing platforms. PLoS ONE 2014;9(8): e104984.

19. Wattam AR, Abraham D, et al. PATRIC, the bacterial bioinformatics database and analysis resource. Nucleic Acids Res. 2014;42(Database issue): D581-D591.

20. Chen L, Zheng D, Liu B, Yang J, Jin Q. VFDB 2016: hierarchical and refined dataset for big data analysis-10 years on. Nucleic Acids Res 2016;44(D1): D694-D697.
21. Pearson $T$, Giffard $P$, et al. Phylogeographic reconstruction of a bacterial species with high levels of lateral gene transfer. BMC Biol 2009;18(7):78.

22. Chirakul S, Norris $\mathrm{MH}$, et al. Transcriptional and post-transcriptional regulation of PenA $\beta$-lactamase in acquired Burkholderia pseudomallei $\beta$-lactam resistance. Sci Rep 2018;13(8):1. PMC6045580.

23. Schweizer HP. Mechanisms of antibiotic resistance in Burkholderia pseudomallei: implications for treatment of melioidosis. Future Microbiology 2012;7(12):1389-1399.

24. Bugrysheva JV, Sue D, et al. Antibiotic resistance markers in Burkholderia pseudomallei strain Bp1651 Identified by genome sequence analysis. Antimicrob Agents Chemother 2017;61(6): e00010-17, e0001017.

25. Schell MA, Ulrich $R L$, et al. Type VI secretion is a major virulence determinant in Burkholderia mallei: Type VI secretion in B. mallei virulence. Mol Microbiol 2007;64(6):1466-1485.

26. Stone JK, DeShazer D, Brett PJ, Burtnick MN. Melioidosis: molecular aspects of pathogenesis. Expert Rev Anti Infect Ther 2014;12(12):1487-1499.

27. Woodman ME, Worth RG, Wooten RM. Capsule influences the deposition of critical complement C3 levels required for the killing of Burkholderia pseudomallei via NADPH-oxidase induction by human neutrophils. PLoS ONE 2012;7(12): e52276.

28. Burtnick MN, Brett PJ, Nair V, Warawa JM, Woods DE, Gherardini FC. Burkholderia pseudomallei Type III secretion system mutants exhibit delayed vacuolar escape phenotypes in raw 264.7 murine macrophages. Infect Immun 2008;76(7):2991-3000.

29. Balder $R$, Lipski $S$, et al. Identification of Burkholderia mallei and Burkholderia pseudomallei adhesins for human respiratory epithelial cells. BMC Microbiol 2010;10(1):250.

30. Saikh KU, Mott TM. Innate immune response to Burkholderia mallei. Curr Opin Infect Dis 2017;30(3):297-302. 